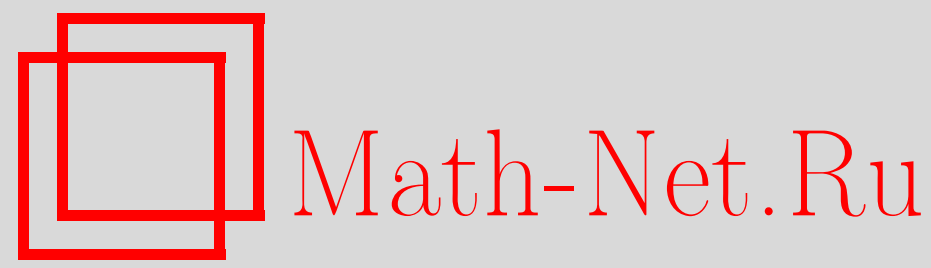

С. Д. Троицкая, О спектре кориолисова оператора в осесимметричных областях с ребрами, Матем. заметки, 1996, том 60, выпуск 2, 304-310

DOI: https://doi.org/10.4213/mzm1830

Использование Общероссийского математического портала MathNet.Ru подразумевает, что вы прочитали и согласны с пользовательским соглашением

http://www . mathnet.ru/rus/agreement

Параметры загрузки:

IP : 52.205 .19 .152

26 апреля 2023 г., 13:11:06

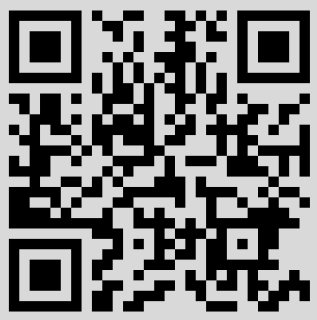




\section{О СПЕКТРЕ КОРИОЛИСОВА ОПЕРАТОРА В ОСЕСИММЕТРИЧНЫХ ОБЛАСТЯХ С РЕБРАМИ}

\section{С. Д. Троицкая}

Пусть $G$ - ограниченная область в $\mathbb{R}^{3}, H$ - гильбертово пространство векторфункций $\vec{U}=\left(u_{1}, u_{2}, u_{3}\right), u_{i} \in L_{2}(G), i=1,2,3$, со скалярным произведением

$$
(\vec{U}, \vec{V})=\int_{G}\left(u_{1} \bar{v}_{1}+u_{2} \bar{v}_{2}+u_{3} \bar{v}_{3}\right) d x d y d z .
$$

В $H$ рассмотрим подпространство $S$, являющееся замыканием линейного многообразия соленоидальных векторов $\left\{\vec{\varphi}=\left(\varphi_{1}, \varphi_{2}, \varphi_{3}\right) \mid \varphi_{i} \in C_{0}^{\infty}(G), i=1,2,3\right.$, $\nabla \cdot \vec{\varphi}=0\}$ и обозначим через $P$ оператор ортогонального проектирования на $S$. Определим линейный оператор $B: S \rightarrow S$ формулой $B \vec{U}=P(\vec{U} \times \vec{k})$, где $\vec{k}=(0,0,1)$. Очевидно, что $B$ ограничен и косоэрмитов. В литературе он часто назьвается гироскопическим или кориолисовым оператором, поскольку уравнение

$$
\frac{\partial \vec{U}}{\partial t}=B \vec{U}, \quad \vec{U}(0)=\vec{U}_{0} \in S
$$

описывает малые колебания идеальной несжимаемой жидкости, целиком заполняющей контейнер $G$ и вращающейся вокруг вертикальной оси $O z$ с постоянной угловой скоростью $\omega=1 / 2$. Изучению этой задачи была посвящена известная работа С. Л. Соболева [1]. Общая теорема о расположении спектра кориолисова оператора (заключающаяся в том, что $\sigma(i B)=[-1,1]$ для произвольной области $G$ ) доказана Дж. В. Ральстоном [2]. Следует отметить, что при этом качественная структура спектра оператора $B$ уже зависит от конфигурации области. В свою очередь, свойства решений задачи Коши (1) (такие, например, как почти периодичность по временно́й переменной $t$ ) тесно связаны именно со структурой спектра этого оператора. Изучению вопроса о почти периодичности решений модельной задачи, соответствующей задаче (1) в случае двух пространственных переменных посвящены работы многих авторов (см. обзоры [3], [4]). Трехмерная задача изучена в значительно меньшей степени. Полностью структура спектра кориолисова оператора исследована только для двух контейнеров - прямого кругового цилиндра и эллипсоида вращения (см. [5]). В обоих этих случаях оператор $i B$ обладает полной системой собственных функций, а множество его собственных значений всюду плотно на отрезке $[-1,1]$, поэтому все решения соответствующей начально-краевой задачи почти периодичны по времени. В [6] доказано существование некоторой тороидальной области, в которой решения уравнения (1) могут быть не почти периодическими.

Ниже рассматриваются области, обладающие осевой симметрией относительно оси вращения. Несложно доказать, что в этом случае пространство $S$ может быть разложено в бесконечную сумму ортогональных друг другу подпространств (будем обозначать их $S_{k}$ ), инвариантных относительно оператора $B$. В настоящей работе доказано, что спектр каждого из операторов $i B_{k}:=\left.i B\right|_{S_{k}}, k=0, \pm 1, \pm 2, \ldots$, являющихся сужениями оператора $i B$ на подпространства $S_{k}$, также совпадает со всем отрезком $[-1,1]$. На основании этого факта с помощью методов работы автоpa [7] (см. также [8], [9]) доказано, что для некоторых осесимметричных областей с 
ребрами (в частности, например, для конуса) спектр оператора $i B_{0}$ является непрерывным на определенных подинтервалах отрезка $[-1,1]$. Это означает, что собственные функции исходного оператора $B$ (если они существуют) не образуют базиса в $S$, и поэтому задача (1) в таких областях обязательно имеет не почти периодические по времени решения.

Далее везде будем предполагать, что $G$ - симметричная относительно оси $O z$ область, ограниченная кусочно-гладкой поверхностью $\partial G$ и удовлетворяющая условию конуса.

Пусть $\widetilde{W}_{2}^{1}:=W_{2}^{1}(G) \ominus\{1\}-$ подпространство пространства С.Л. Соболева $W_{2}^{1}(G)$, ортогональное константам. Пусть $(r, \varphi, t)$ - цилиндрическая система координат, связанная с $(x, y, z): x=r \cos \varphi, y=r \sin \varphi, z=t$, и пусть $D$ - область в плоскости $\varphi=0$, которая заметает $G$ при вращении вокруг оси $O t$. Через $H_{(r)}(P)$ и $\widetilde{W}_{(r)}^{1}(P)$ обозначим пространства, в которые при этой замене координат перейдут соответственно $H$ и $\widetilde{W}_{2}^{1}$. Это гильбертовы пространства классов измеримых функций, заданных на цилиндре $P=D \times(0,2 \pi)$, со скалярными произведениями

$$
\begin{gathered}
(\vec{U}(r, \varphi, t), \vec{V}(r, \varphi, t))_{H_{(r)}(P)}:=\iiint_{P} \sum_{i=1}^{3} u_{i}(r, \varphi, t) \overline{v_{i}(r, \varphi, t)} r d r d \varphi d t \\
(h(r, \varphi, t), g(r, \varphi, t))_{W_{(r)}^{1}(P)} \\
:=\iiint_{P}\left\{h \bar{g}+\frac{\partial h}{\partial r} \frac{\overline{\partial g}}{\partial r}+\frac{\partial h}{\partial t} \frac{\overline{\partial g}}{\partial t}+\frac{1}{r^{2}} \frac{\partial h}{\partial \varphi} \overline{\frac{\partial g}{\partial \varphi}}\right\} r d r d \varphi d t .
\end{gathered}
$$

Обозначим через $L_{(r)}(D)$ и $W_{(k, r)}^{1}(D), k=0, \pm 1, \pm 2, \ldots$, гильбертовы пространства классов измеримых на $D$ функций с нормами

$$
\begin{gathered}
\|f(r, t)\|_{L_{(r)}(D)}^{2}=\iint_{D}|f(r, t)|^{2} r d r d t \\
\|g(r, t)\|_{W_{(k, r)}^{1}(D)}^{2}=\iint_{D}\left\{|g(r, t)|^{2}+\left|\frac{\partial g}{\partial r}\right|^{2}+\left|\frac{\partial g}{\partial t}\right|^{2}+\frac{k^{2}}{r^{2}}|g(r, t)|^{2}\right\} r d r d t
\end{gathered}
$$

соответственно.

ТЕОРема 1. Справедливы представления

$$
\begin{gathered}
\widetilde{W}_{(r)}^{1}(P)=\bigoplus_{k \neq 0, k=-\infty}^{+\infty} W_{(k, r)}^{1}(D) e^{i k \varphi} \bigoplus\left\{W_{(0, r)}^{1}(D) \ominus\{1\}\right\}, \\
H_{(r)}(P)=\bigoplus_{k=-\infty}^{+\infty} H_{(k, r)},
\end{gathered}
$$

где элементы пространств $H_{(k, r)}$ имеют вид

$H_{(k, r)}:=\left\{\left(\begin{array}{ccc}\cos \varphi & -\sin \varphi & 0 \\ \sin \varphi & \cos \varphi & 0 \\ 0 & 0 & 1\end{array}\right)\left(\begin{array}{l}f_{1}(r, t) \\ f_{2}(r, t) \\ f_{3}(r, t)\end{array}\right) e^{i k \varphi}: f_{i}(r, t) \in L_{(r)}(D), i=1,2,3\right\}$, 
и суммы ортогональны относительно скалярных произведений (3) $и$ (2) соответственно.

Обозначим через $S_{(r)}$ пространство, в которое перейдет $S$ при цилиндрической замене координат, через $P_{k}$ - операторы ортогонального (в смысле (2)) проектирования $H_{(r)}$ на подпространства $H_{(k, r)}$, а через $T_{\varphi}$ - ортогональную матрицу в предыдущем выражении.

ТеОРема 2. Справедливы представления

$$
\begin{gathered}
S_{(r)}=\bigoplus_{k=-\infty}^{+\infty} S_{(k, r)}, \\
S_{(k, r)}=\left\{\vec{U}_{k} \in H_{(k, r)}:\left(T_{\varphi}^{-1} \vec{U}, \nabla_{c}\left(p_{k}(r, t) e^{i k \varphi}\right)\right)_{H_{(r)}(P)}=0\right. \\
\left.\forall p_{k}(r, t) \in W_{(k, r)}^{1}(D)\right\},
\end{gathered}
$$

əde

$$
\nabla_{c}:=\left(\frac{\partial}{\partial r}, \frac{1}{r} \frac{\partial}{\partial \varphi}, \frac{\partial}{\partial t}\right)
$$

При этом $P_{k} P=P P_{k}$ для всех $k=0, \pm 1, \pm 2, \ldots$.

Очевидно, что для любого $k$ оператор $P_{S_{(k, r)}}$ ортогонального проектирования $H_{(r)}$ на $S_{(k, r)}$ равен $P_{S_{(k, r)}}=P_{k} P$.

Теорема 3. Пространства $S_{(k, r)}$ приводят оператор B. Для любого $\vec{U} \in S$ имеем $B P_{k} \vec{U}=P_{k} B \vec{U}, k=0, \pm 1, \pm 2, \ldots$.

Мы не приводим доказательств теорем 1-3, поскольку существование инвариантных относительно оператора $B$ подпространств $S_{(k, r)}$ для осесимметричных областей $G$ известно давно (см., например, [10], [11]). В теоремах 1-3 мы просто записали элементы этих пространств в удобной для дальнейших рассуждений форме.

Определим операторы $B_{k}: S_{(k, r)} \rightarrow S_{(k, r)}$ как сужения оператора $B$ на подпространства $S_{(k, r)}: B_{k}:=\left.B\right|_{S_{(k, r)}}, k=0, \pm 1, \pm 2, \ldots$.

TEOPEMA 4. $\sigma\left(i B_{k}\right)=[-1,1], k=0, \pm 1, \pm 2, \ldots$.

ДОКАЗАТЕЛЬСтво. Пусть $\lambda \in(-1,0) \cup(0,1)$. Выберем два действительных числа $\bar{n}_{\text {и }} \bar{m}$ так, чтобы $\bar{m} / \sqrt{\bar{n}^{2}+\bar{m}^{2}}=\lambda$. Ясно, что $\bar{n} \neq 0, \bar{m} \neq 0$. (Для определенности считаем $\bar{n}>0$.) Пусть $\psi \in C_{0}^{\infty}(D): \psi(r, t) \equiv 1$ внутри некоторого квадрата $K_{0} \subset \operatorname{supp} \psi, K_{0}:=\left\{(r, t): r_{0}<r<r_{0}+a_{0}, t_{0}<t<t_{0}+a_{0}\right\}$, и пусть $\rho_{l}:=\psi\left(\sqrt{x^{2}+y^{2}}, z\right) e^{i(\bar{n} l x+\bar{m} l z)}, l=1,2, \ldots$ Тогда из результатов работы [2] следует, что для последовательности вектор-функций

$$
\vec{U}_{l}=\left(\frac{\bar{m} i}{\sqrt{\bar{n}^{2}+\bar{m}^{2}}} \frac{\partial\left(\Delta \rho_{l}\right)}{\partial z}, \frac{\partial\left(\Delta \rho_{l}\right)}{\partial z}, \frac{-\bar{m} i}{\sqrt{\bar{n}^{2}+\bar{m}^{2}}} \frac{\partial\left(\Delta \rho_{l}\right)}{\partial x}-\frac{\partial\left(\Delta \rho_{l}\right)}{\partial y}\right)
$$

существует постоянная $C>0$, не зависящая от номера $l$, такая, что справедлива оценка

$$
\frac{\left\|i B \vec{U}_{l}-\lambda \vec{U}_{l}\right\|_{H}}{\left\|\vec{U}_{l}\right\|_{H}}<\frac{C}{l}, \quad l=1,2, \ldots
$$


Докажем, что для любого $k=0, \pm 1, \pm 2, \ldots$ существуют номер $L(k)<\infty$ и постоянная $C_{k}>0$, не зависящая от $l$, такие, что

$$
\frac{\left\|i B_{k}\left(P_{k} \vec{U}_{l}\right)-\lambda P_{k} \vec{U}_{l}\right\|_{H_{(r)}}}{\left\|P_{k} \vec{U}_{l}\right\|_{H_{(r)}}}<\frac{C_{k}}{l} \quad \forall l \geqslant L(k) .
$$

Пусть

$$
\vec{U}_{l}=\sum_{k=-\infty}^{+\infty} T_{\varphi}\left(\begin{array}{l}
f_{1, k}(r, t) \\
f_{2, k}(r, t) \\
f_{3, k}(r, t)
\end{array}\right) e^{i k \varphi}
$$

Тогда

$$
\begin{aligned}
\left\|P_{k} \vec{U}_{l}\right\|_{H_{(r)}}^{2} & =2 \pi \iint_{D}\left\{\left|f_{1, k}\right|^{2}+\left|f_{2, k}\right|^{2}+\left|f_{3, k}\right|^{2}\right\} r d r d t \geqslant 2 \pi \iint_{D}\left|f_{3, k}\right|^{2} r d r d t \\
& =\iint_{D}\left|\int_{0}^{2 \pi}\left(\vec{U}_{l}, T_{\varphi}\left(\begin{array}{l}
0 \\
0 \\
1
\end{array}\right)\right)_{3} e^{i k \varphi} d \varphi\right|^{2} r d r d t \\
& \geqslant \iint_{K_{0}}\left|\int_{0}^{2 \pi}\left(\vec{U}_{l}, T_{\varphi}\left(\begin{array}{l}
0 \\
0 \\
1
\end{array}\right)\right)_{3} e^{i k \varphi} d \varphi\right|^{2} r d r d t \\
& =\bar{m}^{2} \bar{n}^{2}\left(\bar{m}^{2}+\bar{n}^{2}\right) l^{6} \iint_{K_{0}}\left|\int_{0}^{2 \pi} e^{i(\bar{n} l r \cos \varphi+\bar{m} l t)} e^{i k \varphi} d \varphi\right|^{2} r d r d t,
\end{aligned}
$$

где $(\cdot, \cdot)_{3}$ - эрмитово скалярное произведение в арифметическом пространстве $\mathbb{C}^{3}$, антилинейное по второму аргументу. Имеем (см. [12])

$$
\begin{aligned}
& \iint_{K_{0}}\left|\int_{0}^{2 \pi} e^{i(\bar{n} l r \cos \varphi+\bar{m} l t)} e^{i k \varphi} d \varphi\right|^{2} r d r d t=4 \pi^{2} a_{0} \int_{r_{0}}^{r_{0}+a_{0}} J_{k}^{2}(\bar{n} l r) r d r \\
& =\frac{8 a_{0} \pi}{\bar{n}^{2} l^{2}} \int_{r_{0} \bar{n} l}^{\left(r_{0}+a_{0}\right) \bar{n} l}\left\{\cos ^{2}\left(x-\frac{\pi k}{2}-\frac{\pi}{4}\right)+O\left(\frac{1}{x}\right)\right\} d x,
\end{aligned}
$$

где $J_{k}(z)$ - функция Бесселя. Пусть номер $L_{0}^{\prime}(k)>0$ такой, что $O(1 / x)<1 / 10$ для всех $x \geqslant r_{0} \bar{n} L_{0}^{\prime}(k)$; тогда для $l \geqslant L_{0}^{\prime}(k)$ последнее выражение не меньше, чем

$$
\frac{8 a_{0} \pi}{\bar{n}^{2} l^{2}}\left(\frac{2}{5} a_{0} \bar{n} l-\frac{1}{2}\right) \text {. }
$$

Отсюда следует, что для любого $k=0, \pm 1, \pm 2, \ldots$ существуют номер $L_{0}(k)<\infty$ и постоянная $C_{0}(k)>0$, не зависящая от $l$, такие, что

$$
\left\|P_{k} \vec{U}_{l}\right\|_{H_{(r)}}^{2} \geqslant C_{0}(k) l^{5} \quad \forall l \geqslant L_{1}(k) .
$$

С другой стороны, если $\left\{K_{1}, \ldots, K_{N}\right\}$ - некоторое конечное покрытие $\operatorname{supp} \psi$ квадратами $K_{i}:=\left\{(r, t): r_{i}<r<r_{i}+a_{i}, t_{i}<t<t_{i}+a_{i}\right\}, i=1,2, \ldots, N$, то (см. [2])

$$
\begin{aligned}
& \left\|i B_{k}\left(P_{k} \vec{U}_{l}\right)-\lambda P_{k} \vec{U}_{l}\right\|_{H_{(r)}}^{2}=\left\|P_{k}\left(i B \vec{U}_{l}-\lambda \vec{U}_{l}\right)\right\|_{H_{(r)}}^{2} \\
& \leqslant\left(\bar{n}^{2}+\bar{m}^{2}\right)^{2} l^{4} \sum_{i=1}^{N} C_{i}(k) \iint_{K_{i}}\left|\int_{0}^{2 \pi} e^{i(\bar{n} l r \cos \varphi+\bar{m} l t)} e^{i k \varphi} d \varphi\right|^{2} r d r d t \leqslant C^{\prime}(k) l^{3},
\end{aligned}
$$


откуда следует справедливость оценки (4).

Воспользовавшись результатами работы [1], несложно показать, что если некоторое число $\lambda \in(-1,0) \cup(0,1)$ является собственным значением оператора $B_{k}$, то задача $I_{k}, k=0, \pm 1, \pm 2, \ldots$ :

$$
\begin{gathered}
\frac{\partial^{2} p_{k}}{\partial r^{2}}+\frac{1}{r} \cdot \frac{\partial p_{k}}{\partial r}-\frac{1}{a^{2}} \cdot \frac{\partial^{2} p_{k}}{\partial t^{2}}-\frac{k^{2} p_{k}}{r^{2}}=0 \quad(D), \\
\frac{\partial p_{k}}{\partial r} \cos (\vec{n}, r)-\frac{1}{a^{2}} \frac{\partial p_{k}}{\partial t} \cos (\vec{n}, t)-\left.\frac{k p_{k} \cos (\vec{n}, r)}{\lambda r}\right|_{\partial D \backslash \gamma}=0
\end{gathered}
$$

где $\gamma=G \cap\{r=0\}, a^{2}=\lambda^{2} /\left(1-\lambda^{2}\right)$, при $k= \pm 1, \pm 2, \ldots$ обязательно имеет ненулевое решение $p_{k}(r, t)$ в пространстве $W_{(k, r)}^{1}(D)$, а при $k=0$ - в пространстве $W_{(0, r)}^{1}(D) \ominus\{1\}$. Производные в (5) следует понимать в смысле теории распределений, а граничные условия (6) выполненными почти всюду по линейной мере на $\partial D$ (cм. [7]).

TEOPEMA 5. Пусть

$$
D=\left\{(r, t): 0 \leqslant c<r<d, 0<\varphi_{1}(r)<t<\varphi_{2}(r)\right\}
$$

әде $\varphi_{1}(r), \varphi_{2}(r)$ - гладкие функиии, заданные на $[c, d]$, и либо

$$
\varphi_{1}(c)=\varphi_{2}(c) \quad(c \neq 0), \quad \lim _{r \rightarrow c+0} \varphi_{1}^{\prime}(r) \neq \lim _{r \rightarrow c+0} \varphi_{2}^{\prime}(r),
$$

либо

$$
\varphi_{1}(d)=\varphi_{2}(d), \quad \lim _{r \rightarrow d-0} \varphi_{1}^{\prime}(r) \neq \lim _{r \rightarrow d-0} \varphi_{2}^{\prime}(r),
$$

и пусть $G \subset \mathbb{R}^{3}$ - осесимметричная область, которую заметает $D$ при вращ,ени вокруг оси Ot. Если

$$
M:=\max _{c \leq r \leq d}\left\{\left|\varphi_{i}^{\prime}(r)\right|, i=1,2\right\}<\infty
$$

то для области $G$ на интервалах $\lambda \in\left(-1 / \sqrt{1+M^{2}}, 0\right) \cup\left(0,1 / \sqrt{1+M^{2}}\right)$ спектр оператора $B_{0}$ является чисто непрерывным, т.е. на этих интервалах отсутствуют собственные значения оператора $B_{0}$. (Кривые $\varphi_{i}$, ограничивающие область $D$, могут быть кусочно гладкими, тогда в качестве константы $M$ нужно взять наибольшую из абсолютных величин производньх слева и справа функиий $t=\varphi_{i}(r)$ на отрезке $\left.[c, d].\right)$

ДокАЗАТЕЛЬство. Докажем, что при $\lambda$, принадлежащих указанным интервалам, задача $I_{0}$ имеет только тривиальные решения в пространстве $W_{(0, r)}^{1}(D) \ominus\{1\}$. Для определенности будем считать, что кривые $\varphi_{i}$ пересекаются в точке $d$, т.е. выполнено условие (7). Замена переменных

$$
x=r-a t+a \varphi_{1}(d)-d, \quad y=-r-a t+a \varphi_{1}(d)+d
$$

приводит уравнение (5) при $k=0$ к каноническому виду:

$$
\frac{\partial^{2} p_{0}}{\partial x \partial y}-\frac{1}{2(x-y+2 d)} \cdot \frac{\partial p_{0}}{\partial x}+\frac{1}{2(x-y+2 d)} \cdot \frac{\partial p_{0}}{\partial y}=0 \quad\left(D_{0}\right)
$$


где $D_{0}$ - область в плоскости $O x y$, в которую при этом перейдет $D$, а граничные условия (6) к виду

$$
\left.\left\{\frac{\partial p_{0}}{\partial x} d x-\frac{\partial p_{0}}{\partial y} d y\right\}\right|_{\partial D_{0} \backslash \gamma_{0}}=0
$$

где $\gamma_{0}=\partial D_{0} \cap\{y=x+2 d\}$. Заметим, что область $D_{0}$ ограничена двумя гладкими кривыми, задаваемьми функциями $y=y_{i}(x), x \in I_{i}:=\left(c-a \varphi_{i}(c)+a \varphi_{i}(d)-d, 0\right)$, $i=1,2$, и отрезком прямой $y=x+2 d$. Поскольку $0<\lambda^{2}<1 /\left(1+M^{2}\right)$, то $y_{i}^{\prime}(x)<0 \forall x \in I_{i}, i=1,2$. Кроме того,

$$
y_{1}(0)=y_{2}(0)=0, \quad 0<\lim _{x \rightarrow-0}\left|y_{2}^{\prime}(x)\right|<\lim _{x \rightarrow-0}\left|y_{1}^{\prime}(x)\right| .
$$

На кривых $y_{i}(x)$ граничные условия (9) принимают вид

$$
\frac{\partial p_{0}}{\partial x}-\left.l_{i}^{\prime}(x) \frac{\partial p_{0}}{\partial y}\right|_{y=y_{i}(x)}=0, \quad x \in I_{i}, \quad i=1,2 .
$$

В работе [7] (теоремы 2.1 и 3.2) для некоторого гиперболического уравнения доказано, что для любого $\varepsilon>0$ его решениями в пространстве $W_{2}^{1}\left(D_{0} \cap\{y<x+2 d-\varepsilon\}\right)$, удовлетворяюшими условиям (10), могут быть лишь константы. Наше уравнение (8) отличается от упомянутого лиш знаками коэффициентов, при этом несложно проследить, что те же рассуждения, с помощью которых были доказаны эти теоремы, с незначительньми изменениями проходят и в нашем случае. Поскольку $\varepsilon>0$ произвольно, то отсюда следует, что при $0<\lambda^{2}<1 /\left(1+M^{2}\right)$ решениями задачи $I_{0}$ в пространстве $W_{(0, r)}^{1}(D)$ могут быть только константы. Отбрасьвая их, получаем утверждение теоремы.

Таким образом, в областях $G$, удовлетворяющих условиям теоремы 5 , начально-краевая задача (1) обязательно имеет не почти периодические решения, поскольку собственные векторы оператора $B$ (если такие существуют) не образуют базиса в $S$. Из доказательства теоремы видно, что аналогичный вывод справедлив и для областей, описанных в теореме 3.4 и замечаниях $2.9,2.10,3.5$ работы [7].

Московский государственный институт

Поступило стали и сплавов

16.01 .96

\section{СПИСОК ЦИТИРОВАННОЙ ЛИТЕРАТУРЫ}

1. Соболев С. Л. // Изв. АН СССР. Сер. матем. 1954. Т. 18. №1. С. 3-50. 2. Ralston J. V. // J. Math. Anal. Appl. 1973. V. 44. № 2. Р. 366-383. 3. Александрян Р. А., Березанский Ю. М., Ильин В.А., Костюченко А.Г. // Тр. симпозиума, посвященного 60-летию акад. С. Л. Соболева. М.: Наука, 1970. С. 3-35. 4. Зеленяк Т. И., Капитонов Б. В., Сказка В. В., Фокин М. В. О проблеме С. Л. Соболева в теории малых колебаний вращающейся жидкости. Препринт ВЦ СО АН СССР № 471. Новосибирск, 1983. 5. Barcilon V . // Mathematika. 1968. V. 15. Р. 93-102. 6. Ляшенко А. А. // Докл. АН СССР. 1984. Т. 278. № 4. С. 803-806. 7. Троицкая С. Д. // Изв. РАН. Сер. матем. 1994. Т. 58. № 4. С. 97-124. 8. Троицкал С. Д. // Избр. вопр. алгебры, геометрии и дискретной матем. М.: Изд-во МГУ, 1992. С. 138-147. 9. Троицкая С. Д. // УМН. 1992. Т. 47. № 5. С. 191-192. 10. Соболев С. Л . // Журн. прикл. мех. и техн. физ. 1960. №3. С. 20-55. 11. Гринспэн Х. Теория врашающихся жидкостей. Л.: Гидрометеоиздат, 1975. 12. М. Абрамовиц, И. Стиган (ред. ) Справочник по специальным функциям. М.: Наука, 1979. 